\section{Monitoring the Methanol Conversion Process in H-ZSM-5 using Synchrotron X-ray Powder Diffraction-Mass Spectrometry}

$\dagger \quad$ Dr T.W.B. Lo, Dr L. Ye, Prof S. C. E. Tsang, Wolfson Catalysis Centre, Department of Chemistry, University of Oxford, Oxford, OX1 3QR, UK

$\S \quad$ Dr C. A. Murray, Prof C. C. Tang, Diamond Light Source, Harwell Science and Innovation, Chilton, Didcot, Oxfordshire, OX11 0DE, UK

\# Dr D. Mei, Pacific Northwest National Laboratory, Physical and Computational Sciences Directorate \& Institute for Integrated Catalysis, Richland, WAPO Box 999, USA

$\$ \quad$ These authors contributed equally to this work as joint first authors.

\section{Corresponding Author}

*E-mail: edman.tsang@chem.ox.ac.uk

*Email: donghai.mei@pnnl.gov

5 Supplementary material (SM) is available. 
Using synchrotron X-ray powder diffraction-mass spectrometry in combination with theoretical calculations, we probed the structural changes of adsorbed methanol species in H-ZSM-5 for the first time during catalytic conversion of methanol. Preliminary experimental and computational findings suggested that associative mechanism was the dominant reaction pathway in the early stage of the reaction at $200{ }^{\circ} \mathrm{C}$. Also, we observed a minor contribution from dissociative mechanism of the adsorbed methoxy intermediate species, $\mathrm{CH}_{3}-\mathrm{O}_{z}$ (where $\mathrm{O}_{z}$ is a framework oxygen atom), at Brønsted acid sites. It led to methoxy accumulation during the late stage of the reaction, which was postulated to be the initial step that produced 'hydrocarbon pool' and 'coke' through the formation of C-C bonds.

\section{Graphical Abstract}

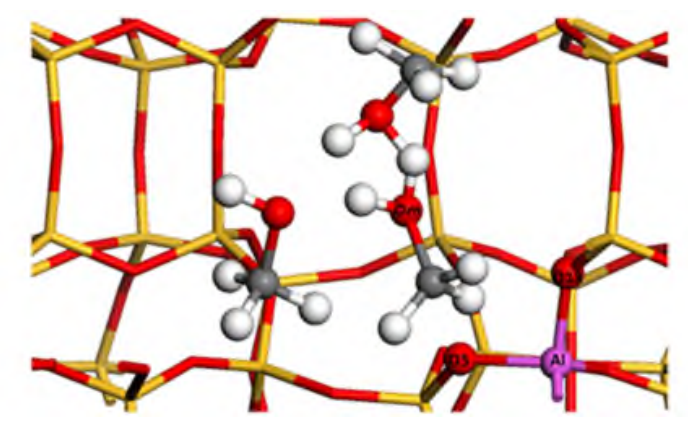

\section{KEYWORDS}

synchrotron X-ray powder diffraction-mass spectrometry $\bullet$ methanol conversion $\cdot$ density functional theory $\bullet \mathrm{H}-\mathrm{ZSM}-5$ zeolites $\bullet$ carbon deposition 


\section{Introduction}

Methanol becomes increasingly important as the building block for chemicals and energy carrier in 'methanol economy' by George A. Olah, the Nobel laureate in Chemistry in 1994. It ultimately helps to replace traditional fossil fuels by utilizing renewable biomass sources. ${ }^{1}$ Although methanol is currently produced from fossil fuels via the syn-gas route, it can also be produced from various biomass sources or $\mathrm{CO}_{2}$ reduction in carbon recycling. ${ }^{1}$ Despite methanol can be directly combusted as a fuel, the general routes to convert methanol to hydrocarbons (MTH) have also recently been attracting more attention as an alternative pathway to crude-oilderived hydrocarbons, such as commodity chemicals (olefins, MTO), aromatics (MTA) and gasoline (MTG), etc. ${ }^{2,3}$ The successful practice of these processes is dependent on the catalyst nature and conditions used. However, one of the most important technical problems is catalyst deactivation by 'carbon' deposition (or so-called 'coke') during methanol conversions. ${ }^{4}$ The catalytic conversions of methanol have been extensively investigated over various acidic catalysts, including zeolites and alumina. ${ }^{5-7}$ However, the prevailing mechanism of how methanol is first activated and the fates of intermediates are still not fully understood. At low temperature of below $270{ }^{\circ} \mathrm{C}$ and high flow rate conditions, methanol dehydration to dimethyl ether (DME) is known to take place favourably. ${ }^{8}$ In fact, the catalytic production of DME is equally important in industry. DME is regarded as a building block for the production of many useful chemicals, including dimethyl sulphate $\left(\left(\mathrm{CH}_{3}\right)_{2} \mathrm{SO}_{4}\right)$ and trimethyloxonium tetrafluoroborate $\left(\left(\mathrm{CH}_{3}\right)_{3} \mathrm{OBF}_{4}\right)$, two common methylating agents, alongside other physical applications, such as freezer spray. ${ }^{9-12}$ Although the reaction route that forms DME from methanol is still debatable, some researchers believe that the organic intermediates as alleged 'hydrocarbon pool' (HCP) in zeolites must be derived from DME or similarly related species. ${ }^{13,14}$ 
Briefly, there are two proposed mechanisms ${ }^{15-18}$ : (i) The dissociative route; methanol is first protonated at a Brønsted acid site (BAS), followed by dehydration which generates a surface methoxy species (SMS). It finally reacts with another incoming methanol. (ii) The associative route; methanol again is first protonated at a BAS. This 'activated' protonated species then combines with another methanol molecule, forming a dimer-like species. Subsequently, the dimer dehydrates to form DME. Recent theoretical modelling efforts by Moses and Nørskov ${ }^{18}$ (in H-ZSM-22), and Grabow et al. ${ }^{19}$ (in H-ZSM-5) have suggested that the associative mechanism dominates at low temperature, whereas the dissociative mechanism prevails at high temperature. Based on comprehensive calculations, Grabow et al. have reported that the 'crossover' temperature between these two mechanisms is zeolite framework and acidity dependent, where the 'crossover' temperature in H-ZSM-5 is around $427{ }^{\circ} \mathrm{C} .{ }^{19}$ Jones and Iglesia further combined theoretical, infra-red spectroscopic and kinetic assessments to show the prevalence of the dissociative mechanism at high reaction temperature. ${ }^{20}$ In fact, the methoxy species $\left(\mathrm{CH}_{3}-\mathrm{O}_{\mathrm{z}}\right.$, where $\mathrm{O}_{\mathrm{z}}$ is a framework $\mathrm{O}$ atom adjacent to the $\mathrm{Al}$ site) has been previously identified by ex-situ spectroscopic techniques such as ${ }^{13} \mathrm{C}$ solid-state $\mathrm{NMR}^{21-23}$ and $\mathrm{FTIR}{ }^{6,24,25}$, which is indicative of the dissociative mechanism. In terms of diffraction techniques, previous works notably by Mentzen, Fitch and van Koningsveld have determined the molecular adsorbate structures in various zeolites. ${ }^{26-30}$ Wragg et al. have investigated the SAPO-34 catalyst in the MTO reaction using high-energy operando synchrotron X-ray powder diffraction (SXRD). The SAPO-34 catalyst expands remarkably and asymmetrically due to the formation of the HCP. ${ }^{31-33}$ Deka et al. have combined SXRD with X-ray absorption fine structure (XAFS) to analyse the $\mathrm{NH}_{3}$-selective catalytic reduction over the structural chemistry of the active $\mathrm{Cu}$ site and $\mathrm{NH}_{3}$ in $\mathrm{Cu}-\mathrm{SSZ}-13$ zeolite. ${ }^{34}$ But there is a general lack of structural studies to verify one mechanism 
from the other during methanol conversion at specific experimental conditions from the structural perspective. It is also possible for the two reactions to take place concurrently, should their activation barriers are both overcome.

We have recently elucidated the structures of organic adsorbates (such as pyridine, ammonia and methanol) even at low coverage in zeolites using SXRD and Rietveld refinement, where zeolites are a class of crystalline but porous template materials. ${ }^{35-37}$ The organic adsorbates reside uniformly over the BAS in our H-ZSM-5 sample. As an alternative to spectroscopic techniques, the bond distances and angles of these organic moieties based on diffraction evidence particularly on the rigid surface BAS of H-ZSM-5 can be measured within experimental errors. Encouraged by these works, we have recently built a high-pressure, low-dead-volume catalytic capillary gas-cell using on-line mass spectrometer with an optimized high-brightness X-ray beam at Beamline I11 in Diamond, UK, to monitor the structure-activity relationship. In this paper, we combined high-resolution SXRD-MS, structural refinement with theoretical calculations to study the catalytic conversion of methanol over H-ZSM-5. The changes of the molecular structure of the adsorbed methanol species on the BAS of fresh H-ZSM-5 during the early stage of the reaction at $200^{\circ} \mathrm{C}$ was probed, where a high activity for DME was prevalent. As the reaction progressed, only small residual activity for DME formation was observed in the late stage (over $10 \mathrm{~h}$ ), largely due to catalyst deactivation. Our structural refinements showed that the methoxy species via the dissociative mechanism is associated with the poly-aromatic 'carbon' in the straight channel of H-ZSM-5, which can be detected at increasing quantity. 


\section{Results and discussion}

100 The H-ZSM-5 sample $\left(\mathrm{H}_{4.48} \mathrm{Al}_{4.48} \mathrm{Si}_{91.52} \mathrm{O}_{192}\right)$ was extensively characterized by NMR 101 spectroscopy, SEM, TEM, and BET (see Figs. S1 and S2 in the SI). In this specific H-ZSM-5 sample, the Al substituted site has been determined specifically at the framework T6 site, whereas the strongest Brønsted acid site at $\mathrm{O} 18$ in our previous work (via loading zeolites with base probe molecules; the zeolite samples were measured with high-resolution SXRD for 1 hour using high-precision multi-analyzer crystal detectors). ${ }^{35}$ It has about one Al per asymmetric unit with the space group of Pnma. The temperature programmed SXRD measurements were first collected, with a ramping rate of $10{ }^{\circ} \mathrm{C} \mathrm{min}^{-1}$ in the capillary reactor under an $\mathrm{N}_{2}$ flow rate of 1

$108 \mathrm{~mL} \mathrm{~min}^{-1}$ at 1 atmosphere using a methanol saturator (kept at RT). The on-line mass spectra 109 (MS) of methanol and DME as the major products (with trace amounts of $\mathrm{CO}, \mathrm{HCHO}$ and $\mathrm{CH}_{4}$ ) 110 were simultaneously monitored. As seen in Fig. 1(a), the slight increase in the methanol 111 concentration from $25-150{ }^{\circ} \mathrm{C}$ is due to the increase in methanol vapour pressure from the 112 saturator near the hot-air blower. Fig. 1(a) also shows the initial dehydration process with an 113 increasing DME concentration from $150{ }^{\circ} \mathrm{C}$ and a corresponding fall in methanol. Also, the 114 corresponding intensity changes of the Bragg peaks at low $2 \theta$ angles can be seen in Fig. 1(b). It 115 is attributed to the corresponding decrease in the population of the adsorbed methanol and 116 increase in thermal vibrations at higher temperatures (see Fig. S3 for kinetic analysis). In 117 general, the lattice of H-ZSM-5 expands slightly at higher temperatures (see Tables S1 for the 118 corresponding crystallographic parameters). 


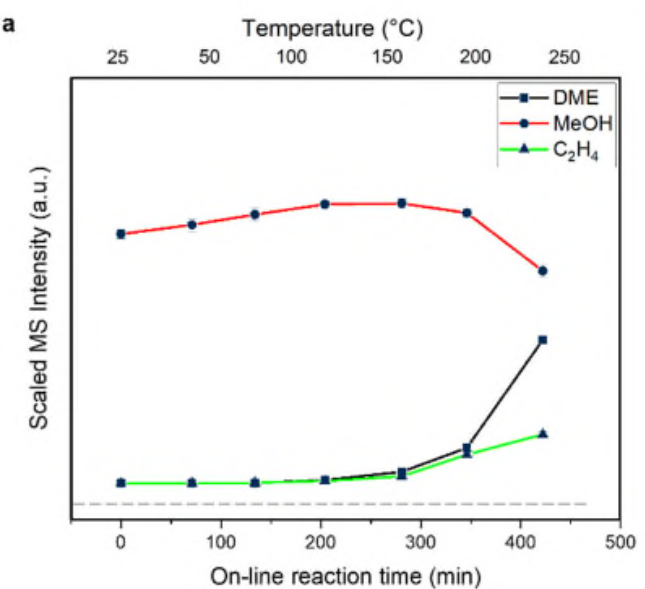

b

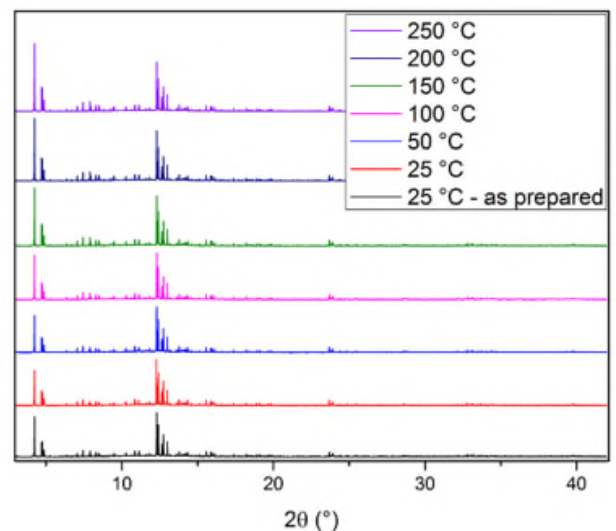

120 Figure 1. On-line synchrotron X-ray powder diffraction (SXRD) and mass spectrometry, with a

121 flowing stream of methanol vapor over H-ZSM-5 (25 to $250{ }^{\circ} \mathrm{C}$ ). (a) On-line mass spectra

122 showing the relative DME and methanol concentrations; noticeable concentration of

123 hydrocarbon products have been detected from $200{ }^{\circ} \mathrm{C}$, showing the initiation of the MTG

124 progress, and (b) SXRD patterns.

125 Fig. 2 shows the selected Rietveld refinement profiles of the on-line SXRD measurements at

12625 and $150{ }^{\circ} \mathrm{C}$ and their corresponding derived crystal structures in an asymmetric unit of a unit

127 cell (the data at other temperatures are summarized in Tables S2). In general, all the SXRD data

128 have been refined well with low R-factors and excellent data fittings. In all the Rietveld derived

129 structures, three $\mathrm{MeOH}$ species are observed in the H-ZSM-5 per asymmetric unit. The position

130 of the $\mathrm{MeOH}$ sites remained basically unchanged upon an average of 10 refinement attempts in

131 manually placing the initial input position at a further distance from the framework, with the

132 derived $\mathrm{O}_{\mathrm{z}^{-}}-\mathrm{O}_{\mathrm{MeOH} 1}$ interatomic distances of $\pm 0.02 \AA$ (which lie within the estimated error

133 values); with the corresponding $\mathrm{R}_{\mathrm{wp}}$ of \pm 0.001 . The verification of the accuracy of the Rietveld

134 refinement processes is summarized in Tables S1-S2 in the SI. Due to the continuous excess feed

135 of methanol into the sample during the online SXRD-MS measurements, the comparatively low 
concentrations of $\mathrm{CO}, \mathrm{CH}_{4}, \mathrm{HCHO}$ and even DME cannot be detected on the BAS. As the concentrations for the formation of DME and any further products are several magnitudes lower than that of methanol adsorption, the majority coverage on the BAS is thus methanol. We acknowledge the increase in thermal motion of the adsorbed species at higher temperatures, therefore the isotropic displacement factors $\left(B_{\text {eq }}\right)$ have been arbitrarily fixed during the 141 refinement (and manually adjusted based on the temperature, see SI/Methods for details).

142 Therefore, similar to our previous work that studies pyridine and ammonia in H-ZSM- $5^{35,36}$, the 143 rigid framework $\mathrm{O}$ atoms (O5 and $\mathrm{O} 18$, adjacent to the only $\mathrm{Al}$ site (T6), previously identified as 144 the $\mathrm{BAS}^{35}$ ) are chosen as the crystallographic pointers in measuring their interatomic distances 145 with the $\mathrm{O}$ atom of the most strongly bound $\mathrm{MeOH}$ species. Note that as $\mathrm{H}$ atoms cannot be 146 directly located by this technique, the $\mathrm{B}_{\text {eq }}$ (isotropic displacement factor) values of the O/C atoms 147 in methanol are arbitrarily fixed. Clustering of $\mathrm{MeOH}$ species within the framework is observed, 148 which agrees with the early work by Forester and Howe. ${ }^{24}$ The closest framework-adsorbate 149 distances between BAS in the sinusoidal-straight cross-channel region and adsorbed methanol is 150 found between $\mathrm{MeOH} 1$ and $\mathrm{O} 18$ and O5, which gives $\mathrm{O} 18-\mathrm{O}_{\mathrm{MeOH} 1}=3.80(4) \AA$ and $\mathrm{O} 5-\mathrm{O}_{\mathrm{MeOH} 1}=$ $1513.85(4) \AA$ A and forms a nearly bidentate mode with O18 and O5. Also, other adsorbed species can 152 be identified and resolved by SXRD under reaction conditions. The uneven negative charge 153 distribution on $\mathrm{O} 5$ (smaller) and $\mathrm{O} 18$ (greater) adjacent to $\mathrm{Al}$ is due to the steric characteristics of 154 H-ZSM-5 that slightly distorts the bidentate morphology. ${ }^{38}$ Comparing to our previous study 155 under a static ex-situ condition, these $\mathrm{O} 18-\mathrm{O}_{\mathrm{MeOH} 1}$ and $\mathrm{O} 5-\mathrm{O}_{\mathrm{MeOH} 1}$ distances are slightly longer. ${ }^{35}$

156 This is presumably due to the dynamic measurement in excess flowing methanol in the present 157 study. We also observe two other $\mathrm{MeOH}$ sites, where their derived interatomic distances are 158 substantially longer than those of $\mathrm{MeOH} 1$. The interatomic distances between MeOH1 with 
$159 \mathrm{MeOH} 2$ and $\mathrm{MeOH} 3$ fall in the regime of H-bonding (between $3.5-3.8 \AA$ ). Thus, a strongly 160 bound protonated methanol $(\mathrm{MeOH} 1)$ and two weakly bound methanol molecules $(\mathrm{MeOH} 2$ and $161 \mathrm{MeOH} 3$ ) inside the H-ZSM-5 cavity are clearly resolved by SXRD under the flowing methanol condition. Despite the increase in thermal vibration (greater errors in measurements), a notable decrease in the site occupancy factor (SOF, the measure of statistical chance of the site occupation) of $\mathrm{MeOH} 1$ from 150 to $250^{\circ} \mathrm{C}$ at a similar rate to the DME formation is observed. The fall in SOF matches with the increasing DME formation (see Table 1). Meanwhile, similar to our previous ammonia desorption study using $\mathrm{SXRD}^{36}$, upon temperature elevation from 25 to $250{ }^{\circ} \mathrm{C}$, the SOF values of $\mathrm{MeOH} 2$ and $\mathrm{MeOH} 3$ also decrease (see Table S4) which reflects a lesser degree of adsorption at higher measurement temperatures even at a continuous feed of methanol. From this study, we only wanted to point out the adsorbed $\mathrm{MeOH}$ detected by SXRD (through the SOF) are likely to be the species consumed and correlated with the formation of DME but with no implication that its disappearance rate equates to the rate of conversion to DME (desorption of $\mathrm{MeOH}$ accounts for the rest). In fact, from the long time-scale of SXRD, we do not think we could reflect the nature of elementary steps taken place on the surface species let

174 alone the differentiation of the rate determining step by this experiment. Besides, the coverage 175 (SOF) must be inversely dependent on the applied temperature which can affect $\mathrm{K}_{\mathrm{ads}}$ (adsorption 176 equilibrium) for this adsorbed species. To substantiate this point, we compared the decrease in $177 \mathrm{MeOH}$ SOF with our previous work that studied H-ZSM-5 adsorbed with pyridine. ${ }^{35}$ It can be 178 seen that even without a continuous flow of substrate vapour, the strongest adsorption site in $\mathrm{H}$ 179 ZSM-5 still displays a similar drop in the SOF at high temperature without chemical reaction. As 180 a result, the temperature effect plays an important role in determining the SOF of adsorbed $181 \mathrm{MeOH}$ in the H-ZSM-5 sample. 
182 Table 1. Derived interatomic distances in comparison from Rietveld refinements and obtained

183 from ab initio molecular dynamics (AIMD) simulations, between the protonated methanol

184 located in the sinusoidal-straight cross-channel region and the framework O18 and O5 atoms.

185 The numbers in the brackets are the estimated standard deviations.

\begin{tabular}{|c|c|c|c|c|c|}
\hline \multirow[t]{2}{*}{$\begin{array}{l}\text { Temperature } \\
\left({ }^{\circ} \mathrm{C}\right)\end{array}$} & \multicolumn{2}{|c|}{$\begin{array}{l}\text { Interatomic distances from } \\
\text { Rietveld refinements (̊) }\end{array}$} & \multirow{2}{*}{$\begin{array}{c}\begin{array}{c}\text { Site occupancy of } \\
\text { MeOH1 }\end{array} \\
\text { SOF of } \mathrm{MeOH} 1\end{array}$} & \multicolumn{2}{|c|}{$\begin{array}{c}\text { Interatomic distances } \\
\text { from AIMD simulations }(\AA)\end{array}$} \\
\hline & $\mathrm{O} 18-\mathrm{O}_{\mathrm{MeOH} 1}$ & $\begin{array}{r}\mathrm{O} 5- \\
\mathrm{O}_{\mathrm{MeOH} 1}\end{array}$ & & $\begin{array}{r}\mathrm{O} 18- \\
\mathrm{O}_{\mathrm{MeOH} 1}\end{array}$ & $\mathrm{O} 5-\mathrm{O}_{\mathrm{MeOH}}$ \\
\hline 25 & $3.71(3)$ & $3.74(3)$ & $0.665(4)$ & 3.85 & 3.75 \\
\hline 50 & $3.72(3)$ & $3.83(3)$ & $0.693(4)$ & 3.95 & 3.95 \\
\hline 100 & $3.63(3)$ & $3.76(3)$ & $0.581(4)$ & 3.95 & 4.05 \\
\hline 150 & $3.41(4)$ & $3.68(4)$ & $0.583(4)$ & 3.45 & 3.55 \\
\hline 200 & $3.40(4)$ & $3.43(4)$ & $0.505(4)$ & 3.45 & 3.55 \\
\hline 250 & $3.39(4)$ & $3.45(4)$ & $0.457(5)$ & 3.35 & 3.55 \\
\hline
\end{tabular}

186 


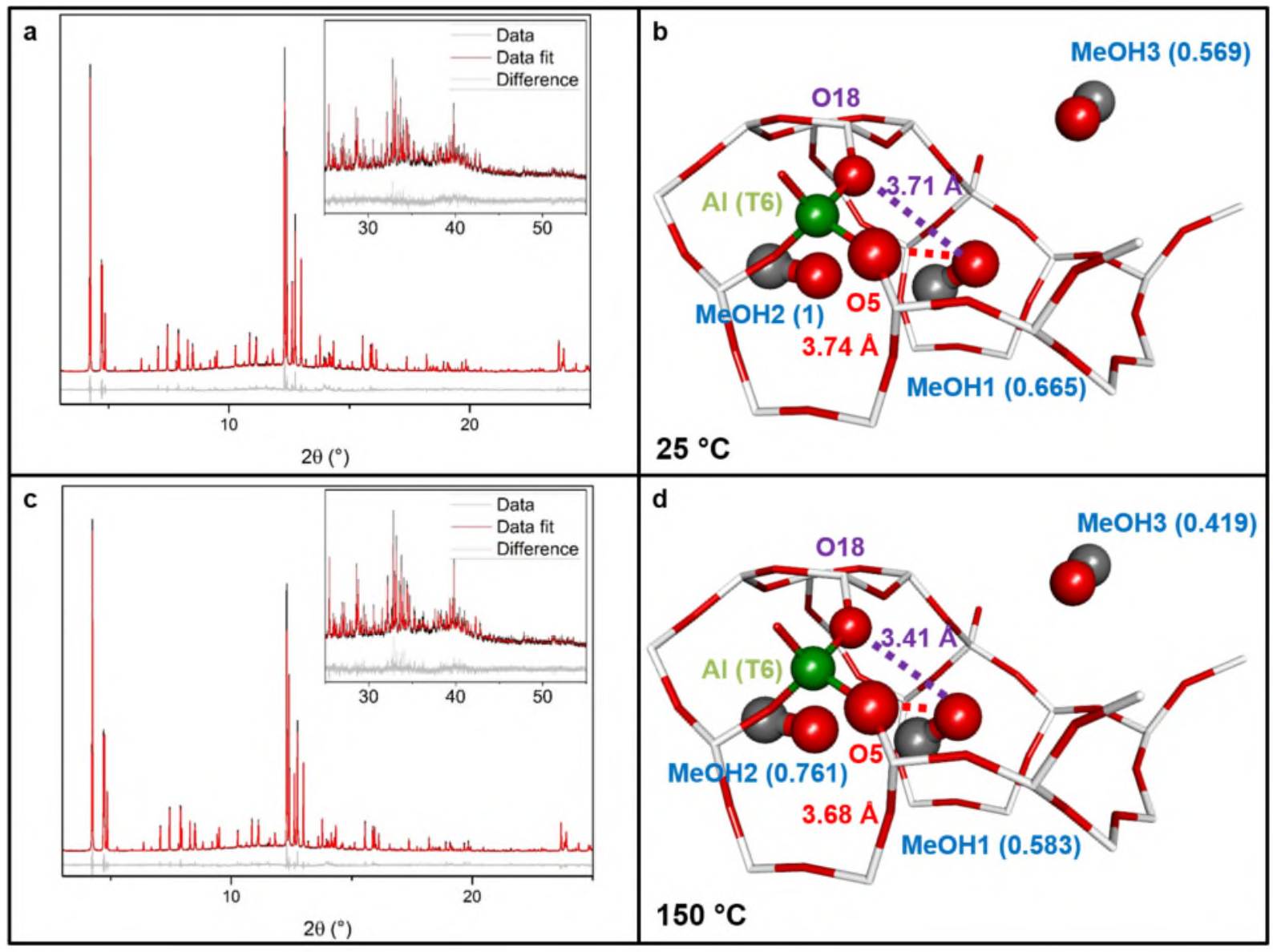

Figure 2. SXRD patterns and Rietveld refinement of the H-ZSM-5 sample with a flowing stream of methanol vapour measured at (a, b) 25 and (c, d) $150{ }^{\circ} \mathrm{C}$. (a, c) The Rietveld refinement profile (At $25{ }^{\circ} \mathrm{C}: R_{\mathrm{wp}}=6.917 \%, \chi^{2}=2.840 ;$ at $\left.150{ }^{\circ} \mathrm{C}: R_{\mathrm{wp}}=6.090 \%, \chi^{2}=2.587\right)$, and $(\mathbf{b}, \mathbf{d})$

191 their corresponding Rietveld derived crystal structures showing an asymmetric unit of a unit cell.

192 The crystallographic and atomic parameters of the complete SXRD measurements are 193 summarized in Tables S3 - S4 in SI, with the corresponding Fourier contrast maps summarized 194 in Fig. S4. The Rietveld refinement profile of H-ZSM-5 without flowing methanol is 195 summarized in Fig. S5 and Table S5. Notice that the adsorbed molecules are described using 196 rigid-body Z-matrices, with the $\mathrm{B}_{\mathrm{eq}}$ (isotropic displacement parameter) of the guest molecules 